\title{
A lectin-based approach to detecting carcinogenesis in breast tissue
}

\author{
GA RAM WI ${ }^{1 *}$, BYUNG-IN MOON ${ }^{2 *}$, HYOUNG JIN KIM ${ }^{1}$, WOOSUNG LIM ${ }^{2}$, \\ ANBOK LEE ${ }^{2}$, JUN WOO LEE ${ }^{2}$ and HONG-JIN KIM ${ }^{1}$ \\ ${ }^{1}$ Laboratory of Virology, College of Pharmacy, Chung-Ang University, Dongjak-Gu, Seoul 156-756; \\ ${ }^{2}$ Breast and Thyroid Cancer Center, Ewha Womans University College of Medicine, \\ Yangcheon-Gu, Seoul 06974, Republic of Korea
}

Received February 21, 2015; Accepted March 15, 2016

DOI: $10.3892 / 01.2016 .4456$

\begin{abstract}
It has been suggested that the diversity of glycosylation structures that form during cancer progression and the sensitivity with which they are able to be detected have great potential for cancer screening. However, the large majority of breast cancer research has instead focused on the development of protein or nucleic acid markers. In the present study, alterations in glycosylation in breast cancer tissue were analyzed using enzyme-linked lectin assays (ELLAs), which have potential for high-throughput screening. Cancer tissues (CCs) and normal tissues (CNs) were collected from women with breast cancer ranging from stage 0 to IIIA. The specimens were divided into two groups, stage 0 -I and stage II-III, and the levels of four types of lectin in stage 0-I and stage II-III CCs and CNs were compared by ELLA. The results demonstrated that, relative to $\mathrm{CNs}$, the $\mathrm{CCs}$ contained significantly enhanced levels of mannosylation (stage 0-I, P<0.001; stage II-III, $\mathrm{P}<0.001$ ), galactosylation (stage $0-\mathrm{I}, \mathrm{P}<0.05$; stage II-III, $\mathrm{P}<0.001$ ), sialylation (stage $0-\mathrm{I}, \mathrm{P}<0.001$; stage II-III, $\mathrm{P}<0.01$ ) and fucosylation (stage $0-\mathrm{I}, \mathrm{P}<0.01$; stage II-III, $\mathrm{P}<0.01$ ). Furthermore, stage II-III CCs had higher levels of mannosylation $(\mathrm{P}<0.05)$ and galactosylation $(\mathrm{P}<0.01)$ than stage $0-\mathrm{I}$ CCs The sensitivity of the ELLA system ranged from 71-100\% when specificity was set at $100 \%$. These results demonstrate that enhanced glycosylation levels identified by ELLA are associated with the development of breast tumors, and provide evidence of the exceptional sensitivity and specificity of the ELLA system in the detection of breast cancer. This approach is anticipated to contribute highly to the development of reliable diagnostic procedures for breast cancer.
\end{abstract}

Correspondence to: Professor Hong-Jin Kim, Laboratory of Virology, College of Pharmacy, Chung-Ang University, 84 Heukseok-Ro, Dongjak-Gu, Seoul 156-756, Republic of Korea E-mail: hongjink@cau.ac.kr

*Contributed equally

Key words: breast cancer, glycosylation, lectin, enzyme-linked lectin assay, mannosylation, galactosylation, sialylation, fucosylation

\section{Introduction}

Breast cancer is the most prevalent cause of mortality in women worldwide, with risk factors that include age, mutation of breast cancer (BRCA)1/BRCA2 genes, age at first full term pregnancy and use of estrogen or progesterone (1). More than 1,300,000 women worldwide are diagnosed with breast cancer each year, and 450,000 women succumb to the disease (2). Standard screening methods include mammography and physical examination (3); however, it is understood that up to $40 \%$ of early breast cancer cases go undetected using these methods (4). Therefore, histological evaluation is currently the gold standard for the detection of breast cancer (5).

Breast cancer is a genetically and clinically heterogeneous disease (6). The histological and clinical outcomes of extensive research indicate that the properties of breast tumors differ significantly between individuals. This has resulted in a novel form of tumor classification that depends on molecular markers (6). Numerous types of molecular markers based on proteins and nucleotide sequences have been studied and developed to aid in determining the diagnosis and prognosis of breast cancer (7). However, only 3 or 4 are currently used in practice, including the estrogen receptor, progesterone receptor and human epidermal growth factor receptor 2 (8). Thus, it is evident that additional makers are required.

Glycosylation involves the formation of complex and heterogeneous structures by stepwise attachment of oligosaccharides and is the most common post-translational modification of proteins and lipids (9). Previous studies have indicated that carcinogenesis is associated with alterations in glycosylation patterns $(10,11)$. The variety of glycan structures and their changes during carcinogenesis have great potential for screening and diagnosis (12). However, to date, the majority studies have focused on identifying protein and nucleotide sequence markers (13).

To analyze glycosylation in clinical biopsy specimens, the majority of previous studies have used mass spectrometry (MS) and high-performance liquid chromatography (HPLC) systems rather than a simple format permitting routine screening (14). The former strategies require specialized techniques and equipment, in addition to lengthy sample preparation. In the present study, alterations in glycosylation patterns were 
Table I. Characteristics of analyzed specimens.

\begin{tabular}{|c|c|c|c|c|c|}
\hline \multirow[b]{2}{*}{ Specimen no. } & \multirow[b]{2}{*}{ Cancer stage } & \multirow{2}{*}{$\begin{array}{c}\text { Patient } \\
\text { age, years }\end{array}$} & \multicolumn{3}{|c|}{ Therapy received } \\
\hline & & & Anti-hormone & Chemotherapy & Radiotherapy \\
\hline \multicolumn{6}{|l|}{ Stage 0-I } \\
\hline 1 & 0 & 44 & $\mathrm{O}$ & & \\
\hline 2 & 0 & 42 & $\mathrm{O}$ & & $\mathrm{O}$ \\
\hline 3 & IA & 62 & $\mathrm{O}$ & & $\mathrm{O}$ \\
\hline 4 & IA & 50 & & $\mathrm{O}$ & \\
\hline \multicolumn{6}{|l|}{ Stage II-III } \\
\hline 5 & IIA & 47 & & $\mathrm{O}$ & \\
\hline 6 & IIA & 70 & & $\mathrm{O}$ & \\
\hline 7 & IIA & 77 & $\mathrm{O}$ & & \\
\hline 8 & IIA & 31 & & $\mathrm{O}$ & \\
\hline 9 & IIB & 39 & & $\mathrm{O}$ & \\
\hline 10 & IIB & 47 & & $\mathrm{O}$ & \\
\hline 11 & IIIA & 44 & & $\mathrm{O}$ & \\
\hline
\end{tabular}

$\mathrm{O}$, indicates treatment received.

investigated as a function of stage of tumor development in breast tissue using an enzyme-linked lectin assay (ELLA) system. Lectins are carbohydrate-binding proteins that recognize specific glycan structures (14). ELLA is the simplest method to analyze lectins; however lectin blotting and lectin microarrays may also be used (15). To date, ELLA systems have been used to investigate a limited number of materials, with purified glycoproteins analyzed most commonly $(16,17)$. There are few previous studies that have analyzed glycan structures in clinical tissue biopsy specimens (18). To the best of our knowledge, the present study demonstrates for the first time that the ELLA system is capable of providing clear evidence of tumor development in tissue biopsy specimens.

\section{Materials and methods}

Clinical samples. A total of 11 breast tissue sets, consisting of paired cancer $(\mathrm{CC})$ and normal $(\mathrm{CN})$ tissues, were obtained from patients who underwent surgical resection at the Ewha Womans University Mokdong Hospital (Yangcheon-Gu, South Korea). The cancer stage, age, and anti-hormone-, chemoand radiotherapy histories of each patient are presented in Table I. Chemotherapy (adriamycin + cyclophosphamide) and hormone therapy (Tamoxen, Tamoxen + Zoladex or Lenara) were conducted following tissue resection, and the stage of each cancer was determined by the tumor-node-metastasis staging system (19). All specimens were immediately frozen and stored at $-80^{\circ} \mathrm{C}$ for subsequent analysis. The present study was conducted with the approval of the Ewha Womans University Mokdong Hospital Institutional Review Board (approval no., EUMC 2014-11-013). Patient samples were obtained with written informed consent from the patients.

Preparation of tissue lysates from biopsies. Tissue lysates were prepared as previously described (18), with modification. Biopsy tissues were cut with scissors and mixed with lysis buffer [100 mM Tris-Cl (pH 7.4; Duchefa Biochemie BV, Haarlem, Netherlands); $150 \mathrm{mM} \mathrm{NaCl}$ (Duchefa Biochemie $\mathrm{BV}$ ); $1 \mathrm{mM}$ ethylenediaminetetraacetic acid-Na (Duchefa Biochemie BV); and 1\% Triton X-100 (USB ${ }^{\circledR}$; affymetrix, Santa Clara, CA, USA)]. The tissues were then disrupted with a Dounce homogenizer (Duran Group GmbH, Mainz, Germany) and centrifuged at $12,000 \mathrm{x}$ g for $10 \mathrm{~min}$ to remove debris and precipitated substances. The supernatants were subsequently collected. Protein concentrations were determined using a Pierce bicinchoninic acid protein assay kit (Thermo Fisher Scientific, Inc., Waltham, MA, USA), with bovine serum albumin serving as a control.

ELLA. Wells of a 96-well microplate (Greiner Bio-One, Frickenhausen, Germany) were coated with 1,600, 800, 400, 200 or $100 \mathrm{ng}$ of tissue lysate protein per well at $4^{\circ} \mathrm{C}$, and blocked with $5 \%$ oxidized bovine serum albumin (BSA; GenDEPOT, Katy, TX, USA) in Tris-buffered saline containing $0.1 \%$ Tween 20 (TBS-T; Duchefa Biochemie BV) for $4 \mathrm{~h}$ at room temperature. The cell lysates were mixed with carbonate buffer ( $\mathrm{pH}$ 9.4) and then incubated for $20 \mathrm{~h}$ at $4^{\circ} \mathrm{C}$ to coat the 96-well plate.. BSA treated with sodium meta-periodate (Sigma-Aldrich, St. Louis, MO, USA) was prepared as previously described (17). Subsequently, the wells were incubated with biotinylated concanavalin A (Con A; catalog no., B-1005), Ricinus communis Agglutinin I (RCA I; catalog no., B-1085), Aleuria aurantia lectin (AAL; catalog no., B-1395) or Maackia amurensis lectin II (MAL II; catalog no., B-1265) (Vector Laboratories, Inc., Burlingame, CA, USA) for $80 \mathrm{~min}$ at $37^{\circ} \mathrm{C}$, followed by poly-horseradish peroxidase (HRP)-conjugated streptavidin (catalog no., N200; Pierce ${ }^{\mathrm{TM}}$; Thermo Fisher Scientific, Inc.) for $40 \mathrm{~min}$ at $37^{\circ} \mathrm{C}$. All lectins were prepared in a reaction buffer (TBS-T containing $0.5 \%$ oxidized BSA) at a concentration of $1 \mu \mathrm{g} / \mathrm{ml}$ lectin. HRP-conjugated streptavidin was diluted 1:5,000 with $0.5 \%$ oxidized BSA in TBS-T. The reactions were developed with 
A

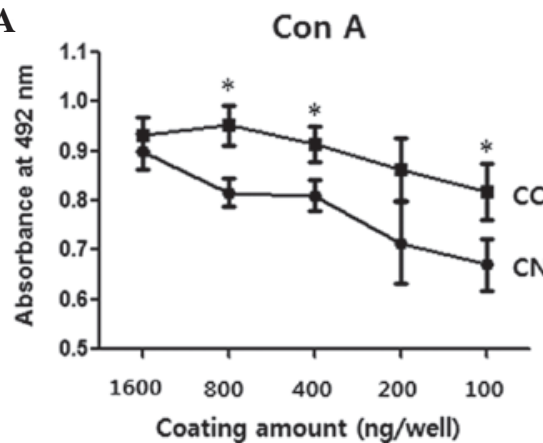

C

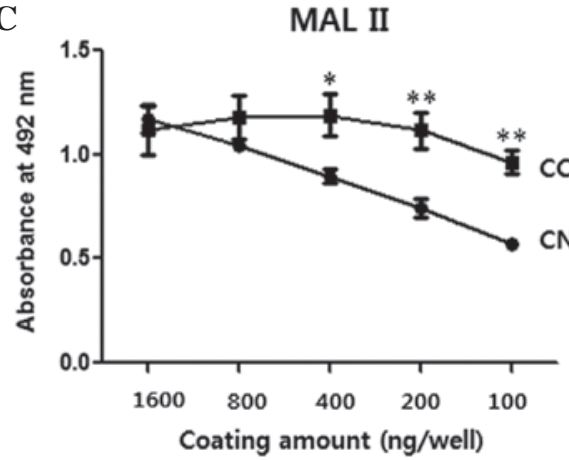

B

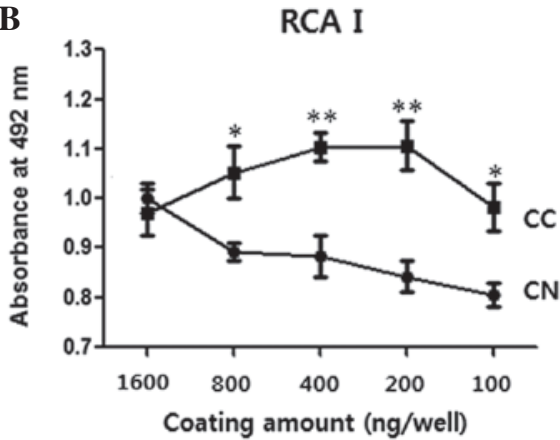

D

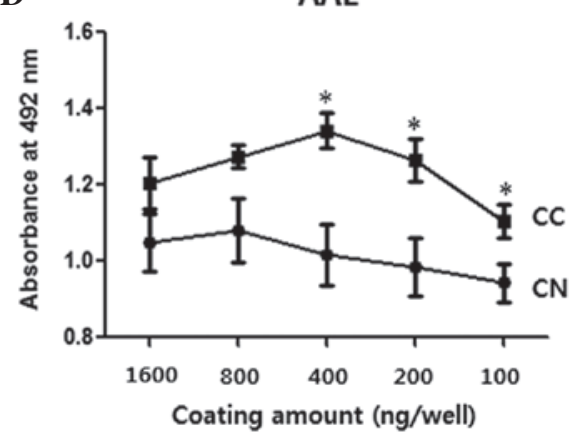

Figure 1. Difference in OD values between CNs and CCs as a function of the amount of protein coating. Each well of a 96-well plate was coated with 1,600, $800,400,200$ or $100 \mathrm{ng}$ of cell lysate protein, and enzyme-linked lectin assays were performed. (A) Con A, (B) RCA I, (C) MAL II and (D) AAL were used to detect mannosylation, galactosylation, sialylation and fucosylation, respectively. Data are presented as the mean \pm standard error. ${ }^{*}<0.05$ and ${ }^{* *} \mathrm{P}<0.01$. OD, optical density; CN, normal tissue; CC, cancer tissue; Con A, concanavalin A; RCA I, Ricinus communis Agglutinin I; MAL II, Maackia amurensis lectin II; AAL, Aleuria aurantia lectin.

$o$-phenylenediamine (Sigma-Aldrich) and optical density (OD) values were measured at $492 \mathrm{~nm}$ using a 96-well plate reader (SUNRISE; Tecan Group Ltd., Männedorf, Switzerland).

Con A affinity chromatography. Con A affinity chromatography was performed as previously described (20), with modification. A $0.8 \times 4.0 \mathrm{~cm}$ Poly-Prep chromatography column (Bio-Rad, Hercules, CA, USA) packed with $0.5 \mathrm{ml}$ Con A Sepharose 4B resin (GE Healthcare Bio-Sciences, Pittsburgh, PA, USA) was equilibrated with binding buffer $[20 \mathrm{mM}$ Tris (pH 7.4; Duchefa Biochemie BV) containing $0.5 \mathrm{M} \mathrm{NaCl}$ (Duchefa Biochemie BV), $1 \mathrm{mM} \mathrm{MgCl}_{2}$ (Sigma-Aldrich) and $1 \mathrm{mM} \mathrm{CaCl}_{2}$ (Sigma-Aldrich)]. Tissue lysates were diluted with binding buffer to $20 \mu \mathrm{g} / \mathrm{ml}$ of protein and loaded into the column. Thereafter, the column was washed with 5 resin-bed volumes of binding buffer. Proteins bound to the Con A resin were eluted with 5 resin-bed volumes of elution buffer [20 mM Tris ( $\mathrm{pH} 7.4$ ) containing $0.5 \mathrm{M} \mathrm{NaCl}$ and $0.5 \mathrm{M}$ methyl- $\alpha$-D-glucopyranoside (Sigma-Aldrich)]. The elution fractions were subsequently examined using the ELLA system.

Lectin blotting. Lectin blots were performed as previously described (18). Samples containing 1,000 ng protein per well were loaded and fractionated on a $12 \%$ acrylamide gel. The fractionated proteins were transferred to a polyvinylidene difluoride membrane (EMD Millipore, Billerica, MA, USA), and blocked with 5\% oxidized BSA in TBS-T for 4 h. The mannosylated proteins were detected with Con A ( $1 \mu \mathrm{g} / \mathrm{ml}$, prepared in $0.5 \%$ oxidized BSA in TBS-T), followed by HRP-conjugated streptavidin (diluted to $1: 5,000$ with $0.5 \%$ oxidized BSA). The membranes were washed three times with TBS-T between reactions, and all reactions were performed at room temperature. The membrane was developed using an enhanced chemiluminescence kit (AbClon, Inc., Seoul, South Korea), and the bands of mannosylated proteins were visualized on X-ray film (Kodak, Rochester, NY, USA).

Statistical analysis. The statistical significance of differences between groups was determined by two-tailed Student's $t$-tests using GraphPad Prism version 5.01 software (GraphPad Software, Inc., La Jolla, CA, USA). P $<0.05$ was considered to indicate a statistically significant difference. Specificity was calculated as follows: Specificity = number of true negatives x 100 / number of true negatives + number of false positives. Sensitivity was calculated by the following: Sensitivity $=$ number of true positives $\times 100 /$ number of true positives + number of false negatives. To determine specificity and sensitivity, the cut-off value was set at the highest value of $\mathrm{CN}$ at each cancer stage, and values higher than the cut-off values were considered as positive for cancer.

\section{Results}

Subgrouping according to cancer stage. Pairs of CN and CC tissues were obtained from each patient with breast cancer. Lysates were prepared from 11 pairs of specimens (Table I) and were used to compare glycosylation levels in the CNs and CCs. The specimens were divided into stage 0 -I and stage II-III, and the glycosylation levels of $\mathrm{CNs}$ and $\mathrm{CCs}$ in each specimen group were measured by ELLA. Stage 0-I tumors were those 

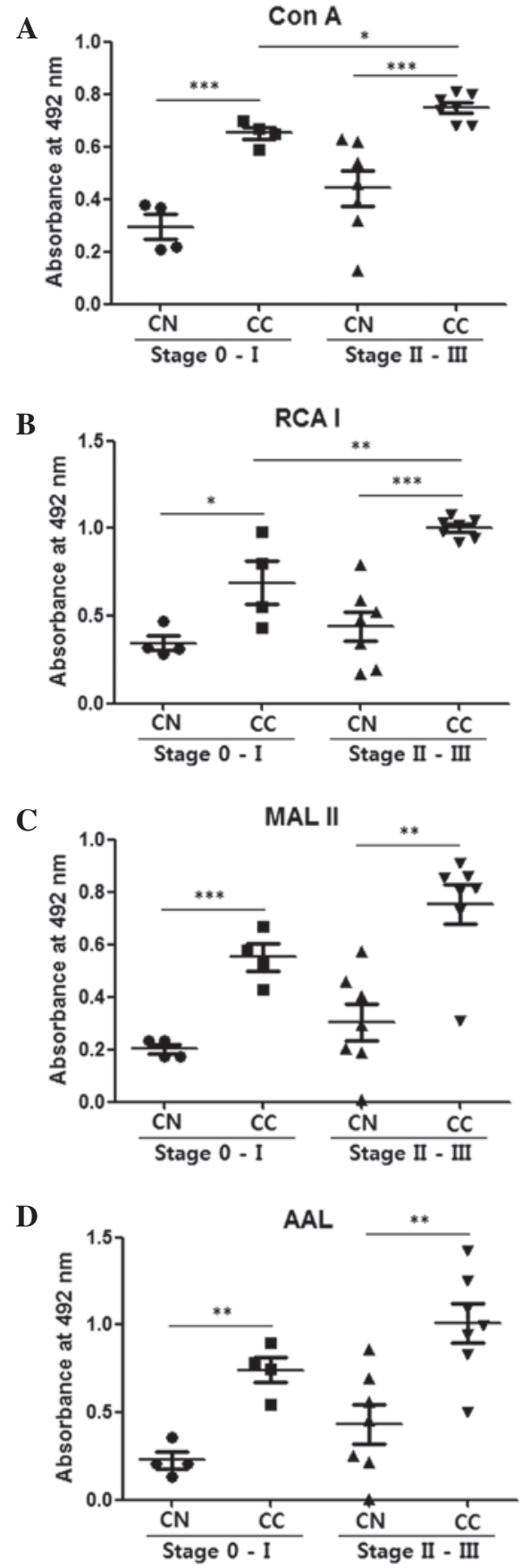

Figure 2. Comparison of CNs and CCs by enzyme-linked lectin assays with 100 ng cell lysate protein coating. (A) Con A, (B) RCA I, (C) MAL II and (D) AAL were used to detect mannosylation, galactosylation, sialylation and fucosylation, respectively. The central line represents the median, and the top and bottom lines represent the 75th and 25th percentiles, respectively. ${ }^{*} \mathrm{P}<0.05,{ }^{* * *} \mathrm{P}<0.01$ and ${ }^{* * *} \mathrm{P}<0.001$. CN (stage $0-\mathrm{I}$ ), $\mathrm{n}=4$; CC (stage $0-\mathrm{I}$ ), $\mathrm{n}=4$; $\mathrm{CN}$ (stage II-III), n=7; CC (stage II-III), n=7. CC, cancer tissue; CN, normal tissue; Con A, concanavalin A; RCA I, Ricinus communis Agglutinin I; MAL II, Maackia amurensis lectin II; AAL, Aleuria aurantia lectin.

not yet metastasized regionally and $<20 \mathrm{~mm}$ in size, while stage II-III tumors were those tending to have spread to axillary lymph nodes or ranging in size from $20-50 \mathrm{~mm}$.

Optimal protein coating for ELLAs. Con A, RCA I, MAL II and AAL recognize mannosylation (preferentially binding

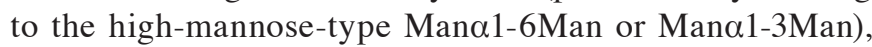
galactosylation (preferentially binding to Gal $\beta 1-4$ GlcNAc), sialylation (preferentially binding to Sia $\alpha 2-3 \mathrm{Gal} \beta 1-4 \mathrm{GlcNAc})$ and fucosylation (preferentially binding to Fuc $\alpha 1-6 \mathrm{GlcNAc}$ or Fuc $\alpha 1-3$ GlcNAc), respectively $(21,22)$. To compare the OD values of the CNs and CCs as a function of the amount of protein used for coating, a 96-well plate was coated with 1,600, $800,400,200$ or $100 \mathrm{ng}$ of protein per well. A total of 4 pairs of $\mathrm{CN}$ and CC (specimens 6, 7, 9 and 10, as presented in Table I) were used for the ELLAs. As shown in Fig. 1, no significant differences were observed between the OD values of $\mathrm{CN}$ and $\mathrm{CC}$ when wells were coated with $1,600 \mathrm{ng}$ of protein $(\mathrm{P}>0.05$; Fig. 1). However, CCs yielded significantly higher values than CNs overall when certain amounts $\leq 800 \mathrm{ng}$ of protein per well were used for coating $(\mathrm{P}<0.05$; Fig. 1$)$. When MAL II was used for the detection of sialylation, the difference between $\mathrm{CNs}$ and $\mathrm{CCs}$ increased with a decreasing amount of protein coating. For the subsequent ELLAs, $100 \mathrm{ng}$ of protein per well was selected (Fig. 2).

Glycosylation levels of CNs and CCs. Fig. 2 compares glycosylation levels in CNs and CCs in the stage 0-I and stage II-III groups. The CCs demonstrated significantly higher values for mannosylation, galactosylation, sialylation and fucosylation compared with the CNs for the stage 0-I and stage II-III groups ( $\mathrm{P}<0.05$; Fig. 2). Stage II-III CCs also demonstrated significantly higher mannosylation $(\mathrm{P}=0.015)$ (Fig. 2A) and galactosylation ( $\mathrm{P}=0.009$; Fig. 2B) levels compared with the stage 0-I CCs. These results indicate that ELLAs have the ability to distinguish stage II-III breast cancer from stage 0-I.

Similar differences were also observed between stage II-III CNs and CCs with fractions from Con A affinity columns enriched for glycoproteins with mannose-type glycans (Fig. 3). These results indicate that CCs have markedly higher levels of glycosylation compared with the equivalent CNs. Banding patterns of $\mathrm{CCs}$ were compared with those of the corresponding CNs following lectin blotting. Overall, the protein bands detected by Con A were stronger in the CCs compared with the CNs (Fig. 4). The blotting results indicated that the CCs generally contained lectin-binding proteins with a mass of $\sim 48 \mathrm{kDa}$, whilst such proteins were absent from the CNs.

Sensitivity and specificity of ELLAs. The sensitivity of each ELLA for discriminating between CCs and CNs when specificity was set at $100 \%$ was investigated (Table II). To set $100 \%$ specificity, the highest OD value of the CNs was used as a cut-off value, and thus the sensitivity of each lectin was evaluated in conditions where there were no false-positives among the CNs. As presented in Table I, Con A had 100\% sensitivity for glycans in stage 0 -I and stage II-III. The other lectins had sensitivities ranging from $71-100 \%$. In addition, it was confirmed that all CCs had higher values than the corresponding CNs, with the exception of one case (specimen 2, RCA I, Table III). These results demonstrate that ELLAs were able to detect CCs with high specificity and sensitivity.

\section{Discussion}

Glycan structures, including sialyl Lewis ${ }^{\mathrm{A}}$, sialyl Lewis ${ }^{\mathrm{X}}$ and Thomsen-Friedenreich (TF) antigen are observed more frequently on the cell membranes of breast cancer tissues compared with normal tissues $(23,24)$. The increased sialyl 
Table II. Sensitivity and specificity of enzyme-linked lectin assays.

\begin{tabular}{|c|c|c|c|c|}
\hline Parameter & Con $\mathrm{A}, \%$ & RCA I, \% & MAL II, \% & AAL, $\%$ \\
\hline \multicolumn{5}{|l|}{ Stage 0-I } \\
\hline Sensitivity & 100 & 75 & 100 & 100 \\
\hline Specificity & 100 & 100 & 100 & 100 \\
\hline \multicolumn{5}{|l|}{ Stage II-III } \\
\hline Sensitivity & 100 & 100 & 86 & 71 \\
\hline Specificity & 100 & 100 & 100 & 100 \\
\hline
\end{tabular}

Con A, concanavalin A; RCA I, Ricinus communis Agglutinin I; MAL II, Maackia amurensis lectin II; AAL, Aleuria aurantia lectin.

\section{A}

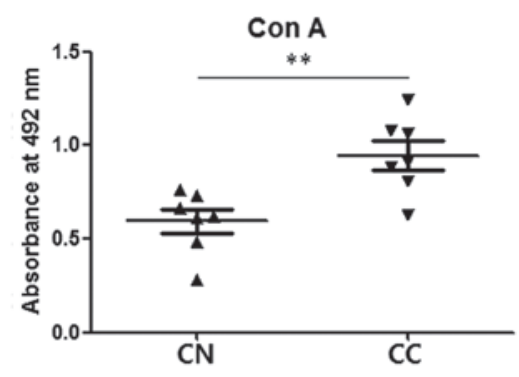

B

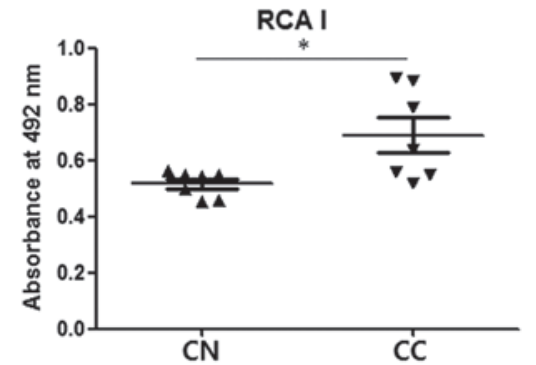

C

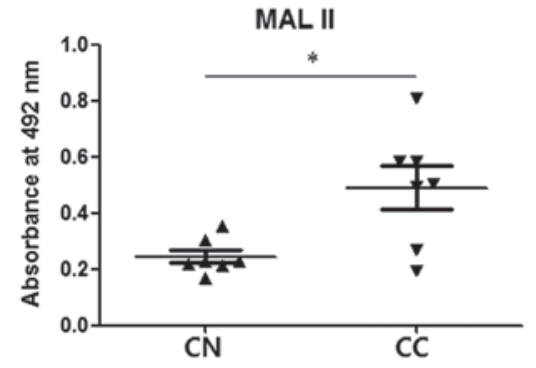

D

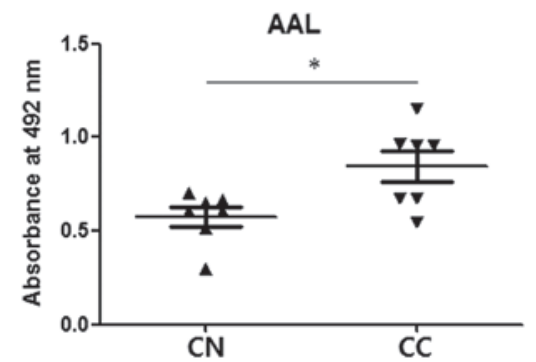

Figure 3. Comparison of elution fractions of CNs and CCs obtained by Con A affinity chromatography. Lysates of stage II-III CNs and CCs were purified by Con A affinity chromatography, and elution fractions were examined by enzyme-linked lectin assay. (A) Con A, (B) RCA I, (C) MAL II and (D) AAL were used to detect mannosylation, galactosylation, sialylation and fucosylation, respectively. The central line represents the median, and the top and bottom lines represent the 75 th and 25 th percentiles, respectively. ${ }^{*} \mathrm{P}<0.05$. $\mathrm{CN}$ (stage II-III), $\mathrm{n}=7$; CC (stage II-III), $\mathrm{n}=7$. CC, cancer tissue; $\mathrm{CN}$, normal tissue; Con A, concanavalin A; RCA I, Ricinus communis Agglutinin I; MAL II, Maackia amurensis lectin II; AAL, Aleuria aurantia lectin.
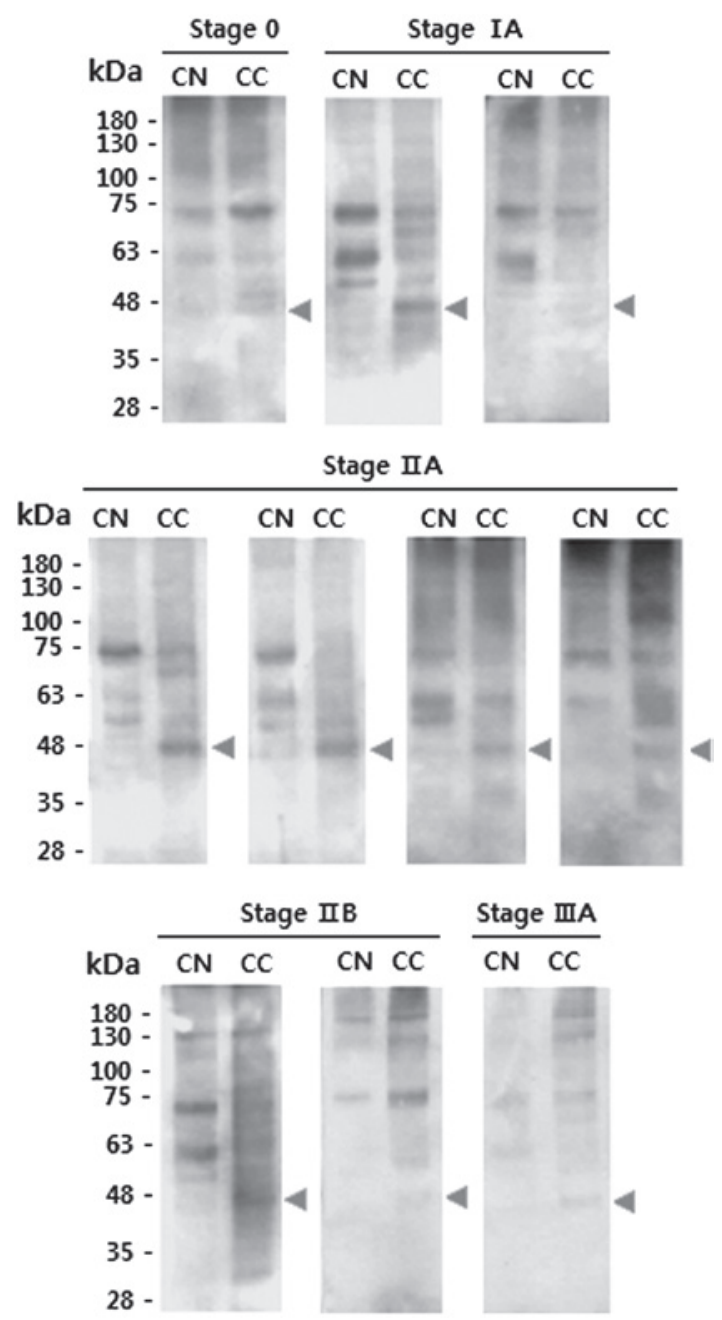

Figure 4. Results of concanavalin A blots comparing CNs and CCs. CNs and $\mathrm{CCs}$ from the same patients were compared. Triangles indicate CC-specific proteins. Specimens used were as follows: Stage 0, no. 1; stage IA, no. 3 and 4; stage IIA, no. 5, 6, 7 and 8; stage IIB, no. 9 and 10; stage IIIA, no. 11 (see Table I). CC, cancer tissue; $\mathrm{CN}$, normal tissue.

Lewis ${ }^{\mathrm{A}}$ and sialyl Lewis ${ }^{\mathrm{X}}$ structures observed in breast cancer are considered to be involved in lymph node metastasis $(22,25)$. Overexpression of sialyl Lewis ${ }^{\mathrm{A}}$, sialyl Lewis ${ }^{\mathrm{X}}$ and TF antigen has also been reported in other types of cancer (26). Similarly, increased terminal sialylation and core fucosylation levels have been detected in several forms of cancer, including liver, colorectal and ovarian cancer (23). Therefore, increased 
Table III. Comparison of OD values of $\mathrm{CN}$ and $\mathrm{CC}$ in individual specimen.

\begin{tabular}{|c|c|c|c|c|c|c|c|c|c|c|c|c|}
\hline \multirow[b]{2}{*}{ Specimen no. } & \multicolumn{3}{|c|}{ Con A } & \multicolumn{3}{|c|}{ RCA I } & \multicolumn{3}{|c|}{ MAL II } & \multicolumn{3}{|c|}{ AAL } \\
\hline & $\mathrm{CN}$ & $\mathrm{CC}$ & $\uparrow / \downarrow$ & $\mathrm{CN}$ & $\mathrm{CC}$ & $\uparrow / \downarrow$ & $\mathrm{CN}$ & $\mathrm{CC}$ & $\uparrow / \downarrow$ & $\mathrm{CN}$ & $\mathrm{CC}$ & $\uparrow / \downarrow$ \\
\hline \multicolumn{13}{|l|}{ Stage 0-I } \\
\hline 1 & 0.37 & 0.67 & $\uparrow$ & 0.31 & 0.98 & $\uparrow$ & 0.17 & 0.58 & $\uparrow$ & 0.14 & 0.78 & $\uparrow$ \\
\hline 2 & 0.38 & 0.65 & $\uparrow$ & 0.47 & 0.43 & $\downarrow$ & 0.23 & 0.43 & $\uparrow$ & 0.36 & 0.55 & $\uparrow$ \\
\hline 3 & 0.21 & 0.70 & $\uparrow$ & 0.32 & 0.80 & $\uparrow$ & 0.18 & 0.67 & $\uparrow$ & 0.21 & 0.75 & $\uparrow$ \\
\hline 4 & 0.22 & 0.59 & $\uparrow$ & 0.28 & 0.55 & $\uparrow$ & 0.23 & 0.53 & $\uparrow$ & 0.21 & 0.90 & $\uparrow$ \\
\hline \multicolumn{13}{|l|}{ Stage II-III } \\
\hline 5 & 0.54 & 0.78 & $\uparrow$ & 0.48 & 0.92 & $\uparrow$ & 0.29 & 0.31 & $\uparrow$ & 0.22 & 0.50 & $\uparrow$ \\
\hline 6 & 0.39 & 0.81 & $\uparrow$ & 0.17 & 1.05 & $\uparrow$ & 0.08 & 0.86 & $\uparrow$ & 0.05 & 0.94 & $\uparrow$ \\
\hline 7 & 0.62 & 0.80 & $\uparrow$ & 0.79 & 0.98 & $\uparrow$ & 0.58 & 0.81 & $\uparrow$ & 0.86 & 0.96 & $\uparrow$ \\
\hline 8 & 0.63 & 0.68 & $\uparrow$ & 0.59 & 1.08 & $\uparrow$ & 0.46 & 0.80 & $\uparrow$ & 0.56 & 1.10 & $\uparrow$ \\
\hline 9 & 0.32 & 0.74 & $\uparrow$ & 0.34 & 1.02 & $\uparrow$ & 0.20 & 0.73 & $\uparrow$ & 0.26 & 0.83 & $\uparrow$ \\
\hline 10 & 0.46 & 0.68 & $\uparrow$ & 0.52 & 0.94 & $\uparrow$ & 0.40 & 0.91 & $\uparrow$ & 0.70 & 1.43 & $\uparrow$ \\
\hline 11 & 0.13 & 0.75 & $\uparrow$ & 0.19 & 1.03 & $\uparrow$ & 0.19 & 0.86 & $\uparrow$ & 0.45 & 1.25 & $\uparrow$ \\
\hline
\end{tabular}

The OD value of each patient's CC was compared with that of the corresponding CN. Values in bold indicate increased OD in CC compared with corresponding CN. OD, optical density; CN, normal tissue; CC, cancer tissue; Con A, concanavalin A; RCA I, Ricinus communis Agglutinin I; MAL II, Maackia amurensis lectin II; AAL, Aleuria aurantia lectin.

glycosylation and the formation of cancer-specific glycan structures are considered as specific features of cancerous tissue $(27,28)$.

Alterations of glycan structures on tumor cell membranes are associated with a poor prognosis and tumor invasiveness (23). Therefore, the majority of studies have focused on histochemical detection of modifications in glycosylation on cell membranes or have used MS and HPLC for similar purposes (29-31). However, these approaches are of limited use for routine and high-throughput analysis. ELLAs have reaction formats similar to those of enzyme-linked immunosorbent assay (ELISA), using 96-well plates and stepwise reactions with target substances. Unlike ELISAs, which are based on antibody reactions and are widely used, defects in lectin reaction conditions have hindered the use of ELLAs for diagnosis $(15)$. Recently, the use of oxidized BSA $(16,18)$ and polymer polyvinyl alcohol (15) has substantially improved the specificity and sensitivity of ELLAs.

To date, there have been only a few attempts to develop ELLAs for routine use in cancer diagnosis via tissue biopsies. In a previous study utilizing ELLA, it was demonstrated that the levels of sialylation and fucosylation in cytoplasmic fractions of cervical cancer tissues were lower than in those of normal cervical tissue, whilst no difference was reported in mannosylation levels (18). However, in the present study, it was observed that levels of mannosylation, galactosylation, fucosylation and sialylation in the CCs were all markedly higher than in the CNs (Fig. 2).

In the current study, the difference between the CNs and CCs was greatest when Con A was used for the ELLA (Fig. 2), and the ELLA system had remarkable sensitivity (71-100\%) despite specificity being set at a maximum value $(100 \%)$. When Con A was used, the ELLAs had $100 \%$ sensitivity and $100 \%$ specificity in discriminating between the CCs and CNs of stages 0-I and II-III. Furthermore, it was confirmed that the Con A protein bands were stronger in the CCs than in the $\mathrm{CNs}$, and the CCs generally contained lectin-binding proteins with a mass of $\sim 48 \mathrm{kDa}$; by contrast, these proteins were absent from the CNs (Fig. 4). Therefore, it is likely that the $48-\mathrm{kDa}$ proteins are specific markers of breast tumor tissue and may serve as potential targets for the treatment of breast cancer.

When Con A was used for affinity chromatography to enrich glycoproteins with the high-mannose form of glycan, the elution fractions from the CCs were observed to have higher levels of mannosylation, galactosylation, sialylation and fucosylation compared with those from the CNs (Fig. 3). This suggests that the glycoproteins with the high-mannose form of glycans in CC tissue also have elevated galactosylation, sialylation and fucosylation levels. A previous study reported that enhanced levels of the high-mannose-type glycans are present in serum from a mouse model of breast cancer and from breast cancer patients (32). Together, these results indicate that the presence of elevated levels of the high-mannose-type glycan is a marker of breast cancer.

As the primary screening system, mammography has contributed to reducing the risk of breast cancer (33). However, histological examination following biopsy, which may cause stress for patients due to its invasive nature, must be performed if a mass is palpable in the breast or an abnormality is observed during a mammogram. Nipple aspiration, originally developed by Papanicolaou et al (34), is an almost non-invasive method for detecting breast cancer, which yields cells from the breast duct lining. Despite the great potential of fine needle nipple aspiration biopsy for breast cancer screening, a high error rate and inability to distinguish invasive cancer from non-invasive cancer have hindered its widespread use (35). Nevertheless, nipple aspiration continues to be used worldwide, particularly in developing countries, due to its low cost. The ELLA system 
used in the present study demonstrated extraordinarily high specificity and sensitivity with only $100 \mathrm{ng}$ of protein required for screening. It is therefore anticipated that ELLA may serve as a complementary system for breast cancer screening, in conjunction with nipple aspiration biopsy.

In conclusion, the current study demonstrated the potential value of the ELLA system as a highly specific and sensitive method for diagnostic screening. Further study of the alterations in glycosylation in breast cancer may provide additional options for reliable diagnosis and prognosis of breast cancer.

\section{Acknowledgements}

This study was supported by a grant from the Korean Health Technology Research and Development Project, Ministry of Health \& Welfare, Republic of Korea (no. HI12C0050; www. htdream.kr/).

\section{References}

1. Donepudi MS, Kondapalli K, Amos SJ and Venkanteshan P Breast cancer statistics and markers. J Cancer Res Ther 10: 506-511, 2014

2. Cancer Genome Atlas Network: Comprehensive molecular portraits of human breast tumours. Nature 490: 61-70, 2012.

3. Kolb TM, Lichy J and Newhouse JH: Comparison of the performance of screening mammography, physical examination and breast US and evaluation of factors that influence them: An analysis of 27, 825 patient evaluations. Radiology 225 165-175, 2002.

4. Alexander H, Stegner AL, Wagner-Mann C, Du Bois GC, Alexander S and Sauter ER: Proteomic analysis to identify breast cancer biomarkers in nipple aspirate fluid. Clin Cancer Res 10 7500-7510, 2004.

5. Ahmed HG: Impact of implementing grading fine needle aspiration cytology in diagnosis of breast cancer amongst sudanese women. Oman Med J 26: 99-103, 2011.

6. Polyak K: Heterogeneity in breast cancer. J Clin Invest 121: 3786-3788, 2011.

7. Esteva FJ and Hortobagyi GN: Prognostic molecular markers in early breast cancer. Breast Cancer Res 6: 109-118, 2004.

8. Malhotra GK, Zhao X, Band $\mathrm{H}$ and Band V: Histological, molecular and functional subtypes of breast cancers. Cancer Biol Ther 10: 955-960, 2010.

9. Grunewald S, Matthijs G and Jaeken J: Congenital disorders of glycosylation: A review. Pediatr Res 52: 618-624, 2002.

10. Li M, Song L and Qin X: Glycan changes: Cancer metastasis and anti-cancer vaccines. J Biosci 35: 665-673, 2010.

11. Reis CA, Osorio H, Silva L, Gomes C and David L: Alterations in glycosylation as biomarkers for cancer detection. J Clin Pathol 63 322-329, 2010.

12. Peracaula R, Barrabés S, Sarrats A, Rudd PM and de Llorens R: Altered glycosylation in tumours focused to cancer diagnosis. Dis Markers 25: 207-218, 2008.

13. van de Vijver MJ: Molecular tests as prognostic factors in breast cancer. Virchows Arch 464: 283-291, 2014.

14. Hirabayashi J: Concept, strategy and realization of lectin-based glycan profiling. J Biochem 144: 139-147, 2008.

15. Thompson R, Creavin A, O'Connell M, O'Connor B and Clarke P: Optimization of the enzyme-linked lectin assay for enhanced glycoprotein and glycoconjugate analysis. Anal Biochem 413: 114-122, 2011.

16. Kim HJ,Lee SJ and Kim HJ: Antibody-based enzyme-linked lectin assay (ABELLA) for the sialylated recombinant human erythropoietin present in culture supernatant. J Pharm Biomed Anal 48 716-721, 2008.
17. Kim HJ, Lee DH, Kim DK, Han GB and Kim HJ: The glycosylation and in vivo stability of human granulocyte-macrophage colony-stimulating factor produced in rice cells. Biol Pharm Bull 31: 290-294, 2008

18. Kim HJ, Kim SC, Ju W, Kim YH, Yin SY and Kim HJ: Aberrant sialylation and fucosylation of intracellular proteins in cervical tissue are critical markers of cervical carcinogenesis. Oncol Rep 31: 1417-1422, 2014.

19. Harris JR: Natural history and staging of breast cancer. In: Diseases of the Breast. Harris JR, Lippman ME, Morrow M and Osborne CK (eds). 1st edition. Lippincott-Raven, Baltimore, PA, pp457-459, 1996.

20. Chen GY, Chen CY, Chang MDT, Matsuura Y and Hu YC: Concanavalin A affinity chromatography for efficient baculovirus purification. Biotechnol Prog 25: 1669-1677, 2009.

21. Clark D and Mao L: Cancer biomarker discovery: Lectin-based strategies targeting glycoproteins. Dis Markers 33: 1-10, 2012.

22. Hirabayashi J, Kuno A and Tateno H: Lectin-based structural glycomics: A practical approach to complex glycans. Electrophoresis 32: 1118-1128, 2011.

23. Renkonen J, Paavonen T and Renkonen R: Endothelial and epithelial expression of sialyl Lewis(x) and sialyl Lewis(a) in lesions of breast carcinoma. Int J Cancer 74: 296-300, 1997.

24. Imai J, Ghazizadeh M, Naito $Z$ and Asano G: Immunohistochemical expression of T, Tn and sialyl-Tn antigens and clinical outcome in human breast carcinoma. Anticancer Res 21 (2B): 1327-1334, 2001.

25. Jeschke U, Mylonas I, Shabani N, Kunert-Keil C, Schindlbeck C, Gerber B and Friese K: Expression of sialyl lewis X, sialyl Lewis A, E-cadherin and cathepsin-D in human breast cancer: Immunohistochemical analysis in mammary carcinoma in situ, invasive carcinomas and their lymph node metastasis. Anticancer Res 25 (3A): 1615-1622, 2005.

26. Christiansen MN, Chik J, Lee L, Anugraham M, Abrahams JL and Packer NH: Cell surface protein glycosylation in cancer. Proteomics 14: 525-546, 2014.

27. Häuselmann I and Borsig L: Altered tumor-cell glycosylation promotes metastasis. Front Oncol 4: 28, 2014.

28. Miyoshi E, Moriwaki K, Terao N, Tan CC, Terao M, Nakagawa T, Matsumoto H, Shinzaki S and Kamada Y: Fucosylation is a promising target for cancer diagnosis and therapy. Biomolecules 2: 34-45, 2012

29. Van Elssen CH, Frings PW, Bot FJ, Van de Vijver KK, Huls MB, Meek B, Hupperets P, Germeraad WT and Bos GM: Expression of aberrantly glycosylated Mucin-1 in ovarian cancer. Histopathology 57: 597-606, 2010.

30. Liu H, Zhang N, Wan D, Cui M, Liu Z and Liu S: Mass spectrometry-based analysis of glycoproteins and its clinical applications in cancer biomarker discovery. Clin Proteomics 11: $14,2014$.

31. Holst S, Stavenhagen K, Balog CI, Koeleman CA, McDonnell LM, Mayboroda OA, Verhoeven A, Mesker WE, Tollenaar RA, Deelder AM and Wuhrer M: Investigations on aberrant glycosylation of glycosphingolipids in colorectal cancer tissues using liquid chromatography and matrix-assisted laser desorption time-of-flight mass spectrometry (MALDI-TOF-MS). Mol Cell Proteomics 12: 3081-3093, 2013.

32. de Leoz ML, Young LJ, An HJ, Kronewitter SR, Kim J, Miyamoto S, Borowsky AD, Chew HK and Lebrilla CB: High-mannose glycans are elevated during breast cancer progression. Mol Cell Proteomics 10: M110.002717, 2011.

33. Weedon-Fekjaer H, Romundstad PR and Vatten LJ: Modern mammography screening and breast cancer mortality: Population study. BMJ 348: g3701, 2014.

34. Papanicolaou GN, Holmquist DG, Bader GM and Falk EA: Exioliative cytology of the human mammary gland and its value in the diagnosis of cancer and other diseases of the breast. Cancer 11: 377-409, 1958.

35. Harigopal M and Chhieng DC: Breast cytology: Current issues and future directions. The Open Breast Cancer Journal 2: 81-89, 2010. 